\title{
LINE OBSERVATIONS OF THE 30-DOR COMPLEX AND N159A5 WITH THE MPE IMAGING NIR SPECTROMETER FAST
}

\author{
A. KRABBE, J. STOREY, V. ROTACIUC, S. DRAPATZ, R. GENZEL \\ Max-Planck-Institut für Physik und Astrophysik \\ Institut für extraterrestrische Physik \\ D-8046 Garching bei München, FR Germany
}

\begin{abstract}
Images with subarcsec spatial resolution in the light of near-infrared atomic (Bry) and molecular hydrogen $\mathrm{H}_{2}(\mathrm{~S}(1) \mathrm{v}=1-0)$ emission lines were obtained for some extended, pointlike objects in the Large Magellanic Cloud (LMC) for the first time. We used the Max-Planck-Institut für extraterrestrische Physik (MPE) near-infrared array spectrometer FAST (image scale $0.8 " /$ pix, spectral resolving power 950) at the ESO/MPI $2.2 \mathrm{~m}$ telescope, La Silla. We present some results on the 30-Dor complex and N159A5.
\end{abstract}

\section{30 Doradus}

The 30-Dor complex around R136 was mosaiced in the light of the Br line $(2.17 \mu \mathrm{m})$ from 27 single images spaced by about 30" covering an area of about 3'x3'. The Bry emission follows roughly the $\mathrm{H} \alpha$ emission, but differences are obvious and can be interpreted in terms of a spatial variation in extinction. Extended emission of molecular hydrogen $\mathrm{H}_{2}$ was discovered for the first time in the LMC about 70" north of R136 with a peak flux of $5 \cdot 10^{-18} \mathrm{~W} / \mathrm{m}^{2} \mathrm{arcsec}^{2}$.

Strong emission of molecular $\mathrm{H}$ (lower flux limit $1 \cdot 10^{-16} \mathrm{~W} / \mathrm{m}^{2}$ ) was detected at the position of a star-like object 45" NE of R136 which is so far classified as an M supergiant. The continuum image taken close to $2.17 \mu \mathrm{m}$ shows a circular source of $\sim 4$ " diameter (seeing was below 1 "). The Bry emission (lower flux limit $1.7 \cdot 10^{-16} \mathrm{~W} / \mathrm{m}^{2}$ ) is also somewhat circular with a diameter in the same range, but indicates high velocity in or out bipolar flow. Pending conclusive interpretation of this object the data that an extended surrounding structure exists.

\section{N159A5}

N159A5 was investigated in detail for the first time by Heydar-Malayeri \& Testor (1982). On their $\mathrm{H} \alpha, \mathrm{H} \beta$, [OIII] plates they discovered a 4" diameter double-peaked nonstellar condensation elongated $\sim 80^{\circ} \mathrm{E}$ of $\mathrm{N}$. Israel \& Koorneef (1988) reported strong Bry and weak $\mathrm{H}_{2}(\mathrm{~S}(1) \mathrm{V}=1-0)$ emission in a 7.5" - 10" aperture. Our near-IR continuum image N159A5 (Fig. 1a) reveals a more complex nucleus and an extended halo of fuzzy structure with $\sim 6$ " diameter. The nucleus, (at least double), elongates (NE-SW), its P.A. corresponding to that of the low-level elongated structure with 50" diameter in a K-band image (Jones et al. 1986). The non-circular shape of the source in their image can be reproduced by convolving our image (note the star to the SE) to their $10^{\text {" spatial }}$ 
resolution. The Bry emission line image (Fig. 1b) with a diameter of $\sim 8$ " looks almost circular and is centred on the SW continuum peak. The $\mathrm{H}_{2}$ emission (Fig. 1c) looks very different and reveals a ring-like structure centred about $2^{2}$ east of $\mathrm{Br} \gamma$. This distance and direction is similar to the double peak found by Heydari-Malayeri \& Testor (1982). The deconvolution of Fig. 1a using the nearby star as a point spread reference function suggests the existence of a multiple component nucleus, a picture also supported by the different geometrical centres of the $\mathrm{Br} \gamma$ and $\mathrm{H}_{2}$ images.
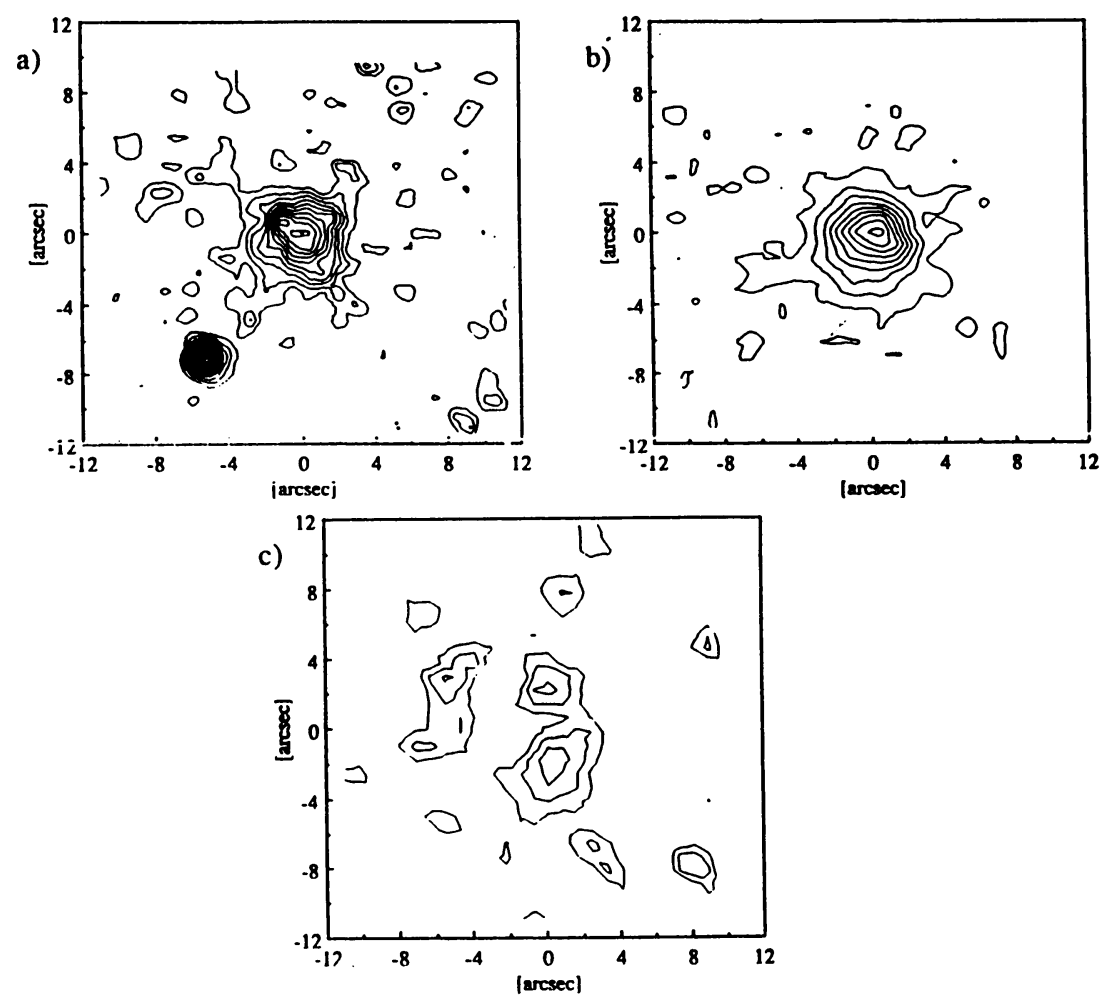

Figure 1. N159A5. a) Continuum image taken with the circular variable filter $(R=50)$ close to $2.12 \mu \mathrm{m}$ at $400 \mathrm{sec}$ total integration time. The contour levels are spaced by $6.7 \cdot 10^{-17} \mathrm{~W} / \mathrm{m}^{2} \operatorname{arcsec}^{2} \mu \mathrm{m}$ with the lowest contour at this level. The total flux at $2.1 \mu \mathrm{m}$ is $1.4 \cdot 10^{-14} \mathrm{~W} / \mathrm{m}^{2} \mu \mathrm{m}$. b) Bry emission at a total integration time of $1200 \mathrm{sec}$ at the position of the line. The contour levels are spaced by $2.5 \cdot 10^{-18}$ $\mathrm{W} / \mathrm{m}^{2}$ arcsec ${ }^{2}$, the lowest contour is $1 \cdot 10^{-18} \mathrm{~W} / \mathrm{m}^{2} \operatorname{arcsec}^{2}$. The total $\mathrm{Br} \gamma$ flux yields $3.3 \cdot 10^{-16} \mathrm{~W} / \mathrm{m}^{2}$. c) The $\mathrm{H}_{2}(\mathrm{~S}(1) \mathrm{v}=1-0)$ emission at a total integration time of $2200 \mathrm{sec}$ on the line position. The contour levels correspond to $9,13,18 \cdot 10^{-19} \mathrm{~W} / \mathrm{m}^{2} \operatorname{arcsec}^{2}$. The total $\mathrm{H}_{2}$ emission is $5.2 \cdot 10^{-17} \mathrm{~W} / \mathrm{m}^{2}$ in a circular aperture of 13 arcsec diameter. The total fluxes in all three images agree well with Israel \& Koornneef (1988).

\section{References}

Heydari-Malayeri, M., Testor, G. (1982), Astron. Astrophys. 111, 116.

Israel, F.P., Koomneef, J. (1988), Astron. Astrophys. 190, 21.

Jones, T.J., Hyland, A.R., Straw, S. et al. (1986), Mon. Not. R. astr. Soc. 219, 603. 